\title{
Kurzzeittherapie mit Venlafaxin statt Lithium?
}

Fragestellung: Die Studie verglich die Effektivität und Sicherheit einer Venlafaxin- versus Lithium-Monotherapie bei Bipolar-II-Patienten mit einer depressiven Episode.

Hintergrund: Der Einsatz von Antidepressiva bei bipolarer Depression ist umstritten. Die Sorge, Patienten damit in eine Manie switchen zu lassen, basiert hauptsächlich auf früheren Studien zum Einsatz von Trizyklika bei einem gemischten $\mathrm{Pa}$ tientengut aus Bipolar-I- und -II-Patienten sowie Patienten mit schizoaffektiver Störung. Studien mit ausschließlich Bipolar-IIPatienten zeigten hingegen eine geringe Switch-Rate bei gutem antidepressiven Effekt. Eine kurze Monotherapie mit Venlafaxin könnte daher einer Therapie mit Lithium überlegen sein, ohne die Switch-Rate zu erhöhen.

Patienten und Methodik: In dieser prospektiven randomisierten Doppelblindstudie wurden jeweils 65 Patienten über zwölf Wochen entweder mit Venlafaxin oder mit Lithium behandelt. Nach Diagnosesicherung über ein SCID-I-Interview (Structured Clinical Interview for DSM-IV Axis I disorders), Ausschluss relevanter Kodiagnosen (Sucht-, Nierenerkrankun-

Amsterdam JD, LorenzoLuaces L, Soeller I et al. Shortterm venlafaxine $v$. lithium monotherapy for bipolar type II major depressive episodes: effectiveness and mood conversion rate. Br J Psychiatry 2016; 208: $359-65$ gen, Nieren- oder Leberinsuffizienz) und bei ausreichender Schwere der Depression (HRSD-17, 17-item Hamilton Rating Scale for Depression $\geq 16$ ) erhielten die Patienten entweder Venlafaxin (37,5 mg mit der
Möglichkeit der Dosistitration auf bis zu $375 \mathrm{mg}$ ) oder Lithium (je nach Lithium-Spiegel 300 - $1.200 \mathrm{mg}$ ). Den verblindeten Behandlern wurden Lithium-Spiegel vorgelegt. Schein-LithiumSpiegel in der Venlafaxin-Gruppe erlaubten es den Behandlern, die Venlafaxin-Dosis gegebenenfalls zu erhöhen.

Primärer Endpunkt war die Response-Rate, definiert als eine Reduktion $u m \geq 50 \%$ im HRSD-17 sowie im CGI/S (clinical global impression severity) ein Score von 1-3 (nicht bis mild depressiv). Sekundäre Endpunkte waren die Remissionsrate (HRSD-17 $\leq$ ), Änderungen im CGI/S und in der YMRS (Young Mania Rating Scale), hypomane oder subsyndromale hypomane Episoden sowie Gewichts- und Blutdruckveränderungen.

Ergebnisse: In der Venlafaxin-Gruppe respondierten 44 Patienten $(67,7 \%)$, in der Lithium-Gruppe $22(34,4 \%, \mathrm{p}=0,0002) .38$ Patienten $(58,5 \%)$ in der Venlafaxin- und $18(28,1 \%)$ in der Lithium-Gruppe erreichten eine Remission ( $p=0,0007)$. Auch im CGI/S zeigte sich in der Venlafaxin-Gruppe eine signifikant größere Reduktion ( $\mathrm{p} \leq 0,0001)$. Dagegen bestanden keine Unterschiede im Hinblick auf Änderungen im YMRS ( $p=0,77)$ und nur minimale, nicht signifikante Gruppenunterschiede bei subsyndromalen hypomanischen Symptomen: 19 Patienten (29,2\%) der Venlafaxin- und 13 (20,3\%) der Lithium-Gruppe zeigten einzelne kurze hypomane Symptome $(\mathrm{p}=0,31)$. Mehr Patienten in der Lithium-Gruppe beendeten wegen fehlender Wirksamkeit die Studie. Die Nebenwirkungsraten waren vergleichbar.

Schlussfolgerungen: Nach zwölf Wochen zeigte Venlafaxin stärkere antidepressive Effekte als Lithium, ohne dabei vermehrt zu hypomanen Symptomen zu führen.

\section{- Kommentar von Alice Engel, Mainz}

\section{Kurztherapie mit Venlafaxin ist antidepressiv wirksam, aber ...}

Es handelt sich hierbei um eine doppelblinde randomisierte klinische Studie zum Vergleich der antidepressiven Wirksamkeit von Venlafaxin im Vergleich zu Lithium bei Bipolar-II-Patienten mit depressiver Episode. Sie erreicht eine hohe Qualität mit 5 von 5 Jadad-Punkten.

Zu den Schwächen der Studie zählt eine fehlende Placebogruppe, auch die kleine Gruppengröße könnte dazu beigetragen haben, dass die Effektivität von Venlafaxin überschätzt wurde. Von den Autoren wurde zusätzlich eine eventuell zu geringe Lithium-Dosis als Grund für die schlechteren ResponseRaten genannt, jedoch lagen die mittleren Spiegelwerte mit $0,94 \mathrm{mmol} / \mathrm{l}$ in einem guten therapeutischen Bereich.

Es zeigten sich wenige hypomane Symptome. Dies kann jedoch durch die kurze Beobachtungsdauer von zwölf Wochen ohne weitere Nachbeobachtungszeit bedingt sein.

Die Studie liefert einen wichtigen Beitrag zur Behandlung von Bipolar-II-Patienten mit schwerer depressiver Episode. Wie auch in den aktuellen Behandlungsleitlinien beschrieben, soll- te Venlafaxin sicher nicht die erste medikamentöse Wahl sein, kann jedoch unter engmaschiger ärztlicher Aufsicht und als Kurztherapie bei therapieresistenten Fällen eine Option darstellen. Größer angelegte multizentrische Studien sind dringend notwendig, um diese ersten positiven Ergebnisse zu replizieren.

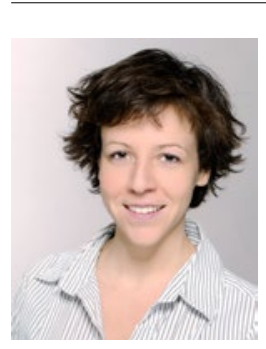

Dr. med. Alice Engel, Mainz

Klinik für Psychiatrie und Psychotherapie, Universitätsmedizin Mainz

E-Mail: alice.engel@unimedizin-mainz.de 